\title{
ON CURVATURE CHARACTERIZATIONS OF SOME HYPERSURFACES IN SPACES OF CONSTANT CURVATURE
}

\author{
Katarzyna Sawicz
}

Communicated by Mileva Prvanović

\begin{abstract}
We investigate curvature properties of pseudosymmetry type of hypersurfaces in semi-Riemannian spaces of constant curvature having the minimal polynomial for the second fundamental tensor of third degree. Among other things we show that the curvature tensor of such hypersurfaces satisfies some condition, which is a generalization of the Roter type equation.
\end{abstract}

\section{Introduction}

A semi-Riemannian manifold $(M, g), n=\operatorname{dim} M \geqslant 3$, is said to be an Einstein manifold if its Ricci tensor $S$ is proportional to the metric tensor $g$, i.e., $S=\frac{\kappa}{n} g$ on $M$. A semi-Riemannian manifold $(M, g), n \geqslant 3$, is called a quasi-Einstein manifold if at every $x \in M$ we have

$$
S=\alpha g+\epsilon w \otimes w, \quad \epsilon= \pm 1,
$$

where $w \in T_{x}^{*} M$ and $\alpha \in \mathbb{R}$. Quasi-Einstein hypersurfaces were studied among others in $[\mathbf{9}]$ and $[\mathbf{1 2}]$, see also references therein. We refer to $[\mathbf{3}]$ for a review of results on quasi-Einstein manifolds. A semi-Riemannian manifold $(M, g), n \geqslant 3$, is Ricci-pseudosymmetric if $R \cdot S=0$ on $M$. Einstein manifolds form a natural subclass of the class of quasi-Einstein manifolds, as well as of the class of Riccisemisymmetric manifolds. We also recall that semisymmetric manifolds, $R \cdot R=0$ on $M[\mathbf{2 2}]$, form a subclass of the class of the Ricci-semisymmetric manifolds. For precise definitions of the symbols used we refer to Sections 2 and 3 of this paper and Sections 2 and 3 of [10] (see also $[\mathbf{2}]$ and $[\mathbf{1 6}]$ ). We mention that the problem of the equivalence of the conditions of semisymmetry $(R \cdot R=0)$ and Ricci-semisymmetry $(R \cdot S=0)$ on hypersurfaces in Euclidean spaces, named the

2000 Mathematics Subject Classification: Primary 53B20, 53B25; Secondary 53C25.

Key words and phrases: warped product, hypersurface, pseudosymmetry type condition, the Roter type equation.

Research supported by Technical University of Czestochowa (Poland). 
problem of P. J. Ryan, lead to considerations of quasi-Einstein hypersurfaces (see e.g., $[\mathbf{1}],[\mathbf{8}])$. An extension of the class of semisymmetric manifolds form also pseudosymmetric manifolds (see e.g., [3, Sections 3 and 4]). A semi-Riemannian manifold $(M, g), n \geqslant 3$, is said to be pseudosymmetric if, at every point of $M$, the tensors $R \cdot R$ and $Q(g, R)$ are linearly dependent. This is equivalent to

$$
R \cdot R=L_{R} Q(g, R)
$$

on $\mathcal{U}_{R}=\left\{x \in M \mid R-\frac{\kappa}{(n-1) n} G \neq 0\right.$ at $\left.x\right\}$, where $L_{R}$ is some function on $\mathcal{U}_{R}$. Further, a semi-Riemannian manifold $(M, g), n \geqslant 3$, is said to be Riccipseudosymmetric [3] if, at every point of $M$, the tensors $R \cdot S$ and $Q(g, S)$ are linearly dependent. This is equivalent to

$$
R \cdot S=L_{S} Q(g, S)
$$

on $\mathcal{U}_{S}=\left\{x \in M \mid S-\frac{\kappa}{n} g \neq 0\right.$ at $\left.x\right\}$, where $L_{S}$ is some function on $\mathcal{U}_{S}$. The class of Ricci-pseudosymmetric manifolds is an extension of the class of Riccisemisymmetric manifolds, as well as of the class of pseudosymmetric manifolds $[3]$.

Let $H$ be the second fundamental tensor of a hypersurface $M$ immersed isometrically in a semi-Riemannian space of constant curvature $N_{s}^{n+1}(c)$, with signature $(s, n+1-s), n \geqslant 4$, where $c=\frac{\widetilde{\kappa}}{n(n+1)}$ and $\widetilde{\kappa}$ denotes the scalar curvature of the ambient space. Let $\mathcal{U}_{H} \subset M$ be the set of all points at which the tensor $H^{2}$ is not a linear combination of $H$ and the metric tensor $g$ of $M$. In this paper we will investigate hypersurfaces $M$ satisfying on $\mathcal{U}_{H} \subset M$

$$
H^{3}=\operatorname{tr}(H) H^{2}+\psi H+\rho g,
$$

where $\psi$ and $\rho$ are some functions on $\mathcal{U}_{H}$. Examples of hypersurfaces satisfying (1.4) with $\rho=0$ are presented [10] (see also [14]). It is known that such hypersurfaces are Ricci-pseudosymmetric (see e.g., [5]). Recently examples of hypersurfaces of dimension $n \geqslant 5$, satisfying (1.4), with nonzero function $\rho$, were found in [21]. We refer to $[\mathbf{1 7}]$ for results related to hypersurfaces in spaces of constant curvature for which the tensor $H^{2}$ is a linear combination of $H$ and $g$.

Let $M$ be a hypersurface in $N_{s}^{n+1}(c), n \geqslant 4$. We have

$$
R \cdot S=\frac{\widetilde{\kappa}}{n(n+1)} Q(g, S)
$$

at $x \in \mathcal{U}_{H} \subset M$ if and only if $H^{3}=\operatorname{tr}(H) H^{2}+\alpha H, \alpha \in \mathbb{R}$, at this point [5, Theorem 3.1].

Let $U_{1} \subset \mathcal{U}_{H} \subset M$ the set of all points at which $R \cdot S \neq \frac{\widetilde{\kappa}}{n(n+1)} Q(g, S)$. We note that if (1.4) holds at $x \in \mathcal{U}_{H}$, then $x \in U_{1} \subset \mathcal{U}_{H}$ if and only if $\rho \neq 0$ at this point. We also mention that (1.4) implies (see e.g., Proposition 3.2(i))

$$
R \cdot S=\frac{\widetilde{\kappa}}{n(n+1)} Q(g, S)+\rho Q(g, H) .
$$

In $\left[\mathbf{2 0}\right.$, Theorem 5.1] it is proved that (1.4) is equivalent on $U_{1}$ to

$$
C \cdot R=\frac{n-3}{n-2} Q(S, R)+\alpha_{1} Q(g, R)+\alpha_{2} Q(S, G),
$$




$$
\alpha_{1}=\frac{1}{n-2}\left(\frac{\kappa}{n-1}+\varepsilon \psi-\frac{\left(n^{2}-3 n+3\right) \widetilde{\kappa}}{n(n+1)}\right), \quad \alpha_{2}=-\frac{(n-3) \widetilde{\kappa}}{(n-2) n(n+1)} .
$$

(1.6) is a condition of pseudosymmetry type. We refer to $[\mathbf{3}]$ for a survey of results related to manifolds, and in particular to hypersurfaces satisfying pseudosymmetry type conditions. Hypersurfaces $M$ in $N_{s}^{n+1}(c), n \geqslant 4$, having the tensor $R \cdot C$ expressed by a linear combination of the tensors $Q(S, R), Q(g, R)$ and $Q(S, G)$, were investigated in [16] (see also [10]). Among other things in [16] it was shown that such hypersurfaces $M$ satisfy (1.5) on $\mathcal{U}_{H} \subset M$ and in a consequence, they are Ricci-pseudosymmetric. In [14, Proposition 2.1] it is proved that every hypersurface $M$ in $N_{s}^{5}(c)$ satisfying (1.4) is pseudosymmetric. Precisely, on $\mathcal{U}_{H} \subset M$ we have

$$
R \cdot R=\frac{\widetilde{\kappa}}{n(n+1)} Q(g, R)
$$

and $\operatorname{rank} H=2$. The last relation implies $H^{3}=\operatorname{tr}(H) H^{2}+\psi H$, for some function $\psi$ on $\mathcal{U}_{H}$ (see [6, Lemma 2.1]).

We mention that hypersurfaces $M$ in $N_{s}^{n+1}(c), n \geqslant 4$, satisfying (1.4) and some curvature conditions, named Ricci-type equations, were recently investigated in $[\mathbf{1 9}]$.

We recall that the curvature tensor $R$ of a Roter type manifold $(M, g), n \geqslant 4$, is expressed on $\mathcal{U}_{C} \cap \mathcal{U}_{S} \subset M$ by a linear combination of the tensors $S \wedge S, g \wedge S$ and $g \wedge g$, i.e., (2.5) holds on this set. Our investigations lead to a new condition for the curvature tensor $R$. We prove (see Theorem 3.2) that the tensor $R$ of a hypersurface $M$ in $N_{s}^{n+1}(c), n \geqslant 4$, satisfying (1.4) is expressed on $U_{1} \subset \mathcal{U}_{H} \subset M$ by a linear combination of the tensors $S^{2} \wedge S^{2}, S \wedge S^{2}, g \wedge S^{2} S \wedge S, g \wedge S$ and $g \wedge g$ (see 3.13). Clearly, (2.5) is a special case of (3.13). In Section 3 we also prove (see Theorem 3.1) that on $U_{1} \subset \mathcal{U}_{H} \subset M$ of a hypersurface $M$ in $N_{s}^{n+1}(c), n \geqslant 4,(1.4)$ is equivalent to (3.6).

In the last section we investigate hypersurfaces $M$ in $N_{s}^{n+1}(c), n \geqslant 4$, satisfying (1.4), which are locally warped products. Among other things we prove (see Theorems 4.2 and 4.3) that under some additional assumptions we have $\rho=0$. We also present curvature properties of such hypersurfaces (see Theorem 4.3).

The author would like express her thanks to Professor Ryszard Deszcz for his help during the preparation of this paper.

\section{Preliminaries}

Throughout this paper all manifolds are assumed to be connected paracompact manifolds of class $C^{\infty}$. Let $(M, g)$ be an $n$-dimensional, $n \geqslant 3$, semi-Riemannian manifold and let $\nabla$ be its Levi-Civita connection and $\mathfrak{X}(M)$ the Lie algebra of vector fields on $M$. We define on $M$ the endomorphisms $X \wedge_{A} Y$ and $\mathcal{R}(X, Y)$ of $\mathfrak{X}(M)$ by

$$
\begin{aligned}
\left(X \wedge_{A} Y\right) Z & =A(Y, Z) X-A(X, Z) Y, \\
\mathcal{R}(X, Y) Z & =\nabla_{X} \nabla_{Y} Z-\nabla_{Y} \nabla_{X} Z-\nabla_{[X, Y]} Z,
\end{aligned}
$$

respectively, where $A$ is a symmetric $(0,2)$-tensor on $M$ and $X, Y, Z \in \mathfrak{X}(M)$. The Ricci tensor $S$, the Ricci operator $\mathcal{S}$ and the scalar curvature $\kappa$ of $(M, g)$ are defined 
by

$$
S(X, Y)=\operatorname{tr}\{Z \rightarrow \mathcal{R}(Z, X) Y\}, \quad g(\mathcal{S} X, Y)=S(X, Y), \quad \kappa=\operatorname{tr} \mathcal{S},
$$

respectively. The endomorphism $\mathcal{C}(X, Y)$ is defined by

$$
\mathcal{C}(X, Y) Z=\mathcal{R}(X, Y) Z-\frac{1}{n-2}\left(X \wedge_{g} \mathcal{S} Y+\mathcal{S} X \wedge_{g} Y-\frac{\kappa}{n-1} X \wedge_{g} Y\right) Z .
$$

Let $\mathcal{B}(X, Y)$ be a skew-symmetric endomorphism of $\mathfrak{X}(M)$ and let $B$ be a $(0,4)$ tensor associated with $\mathcal{B}(X, Y)$ by

$$
B\left(X_{1}, X_{2}, X_{3}, X_{4}\right)=g\left(\mathcal{B}\left(X_{1}, X_{2}\right) X_{3}, X_{4}\right),
$$

where $X_{1}, X_{2}, \ldots \in \mathfrak{X}(M)$. The tensor $B$ is said to be a generalized curvature tensor if

$$
\begin{gathered}
B\left(X_{1}, X_{2}, X_{3}, X_{4}\right)+B\left(X_{2}, X_{3}, X_{1}, X_{4}\right)+B\left(X_{3}, X_{1}, X_{2}, X_{4}\right)=0 \\
B\left(X_{1}, X_{2}, X_{3}, X_{4}\right)=B\left(X_{3}, X_{4}, X_{1}, X_{2}\right) .
\end{gathered}
$$

We define the $(0,4)$-tensor $G$, the Riemann-Christoffel curvature tensor $R$ and the Weyl conformal curvature tensor $C$ by

$$
\begin{aligned}
& G\left(X_{1}, X_{2}, X_{3}, X_{4}\right)=g\left(\left(X_{1} \wedge_{g} X_{2}\right) X_{3}, X_{4}\right), \\
& R\left(X_{1}, X_{2}, X_{3}, X_{4}\right)=g\left(\mathcal{R}\left(X_{1}, X_{2}\right) X_{3}, X_{4}\right), \\
& C\left(X_{1}, X_{2}, X_{3}, X_{4}\right)=g\left(\mathcal{C}\left(X_{1}, X_{2}\right) X_{3}, X_{4}\right),
\end{aligned}
$$

respectively. These tensors are generalized curvature tensors.

Let $\mathcal{B}(X, Y)$ be a skew-symmetric endomorphism of $\mathfrak{X}(M)$ and let $B$ be the tensor defined by (2.1). We extend the endomorphism $\mathcal{B}(X, Y)$ to derivation $\mathcal{B}(X, Y)$. of the algebra of tensor fields on $M$, assuming that it commutes with contractions and $\mathcal{B}(X, Y) \cdot f=0$, for any smooth function $f$ on $M$. Now for a $(0, k)$-tensor field $T, k \geqslant 1$, we define the $(0, k+2)$-tensor $B \cdot T$ by

$$
\begin{aligned}
& (B \cdot T)\left(X_{1}, \ldots, X_{k} ; X, Y\right)=(\mathcal{B}(X, Y) \cdot T)\left(X_{1}, \ldots, X_{k}\right) \\
& \quad=-T\left(\mathcal{B}(X, Y) X_{1}, X_{2}, \ldots, X_{k}\right)-\cdots-T\left(X_{1}, \ldots, X_{k-1}, \mathcal{B}(X, Y) X_{k}\right) .
\end{aligned}
$$

In addition, if $A$ is a symmetric $(0,2)$-tensor, then we define the $(0, k+2)$-tensor $Q(A, T)$ by

$$
\begin{aligned}
& Q(A, T)\left(X_{1}, \ldots, X_{k} ; X, Y\right)=\left(X \wedge_{A} Y \cdot T\right)\left(X_{1}, \ldots, X_{k}\right) \\
& \quad=-T\left(\left(X \wedge_{A} Y\right) X_{1}, X_{2}, \ldots, X_{k}\right)-\cdots-T\left(X_{1}, \ldots, X_{k-1},\left(X \wedge_{A} Y\right) X_{k}\right) .
\end{aligned}
$$

In this manner we obtain the $(0,6)$-tensors $B \cdot B$ and $Q(A, B)$. Setting in the above formulas $\mathcal{B}=\mathcal{R}$ or $\mathcal{B}=\mathcal{C}, T=R$ or $T=C$ or $T=S, A=g$ or $A=S$, we get the tensors $R \cdot R, R \cdot C, C \cdot R, R \cdot S, Q(g, R), Q(S, R), Q(g, C)$ and $Q(g, S)$. For symmetric $(0,2)$-tensors $E$ and $F$ we define their Kulkarni-Nomizu product $E \wedge F$ by

$$
\begin{aligned}
(E \wedge F)\left(X_{1}, X_{2}, X_{3}, X_{4}\right)= & E\left(X_{1}, X_{4}\right) F\left(X_{2}, X_{3}\right)+E\left(X_{2}, X_{3}\right) F\left(X_{1}, X_{4}\right) \\
& -E\left(X_{1}, X_{3}\right) F\left(X_{2}, X_{4}\right)-E\left(X_{2}, X_{4}\right) F\left(X_{1}, X_{3}\right) .
\end{aligned}
$$


Clearly, the tensors $R, C, G$ and $E \wedge F$ are generalized curvature tensors. For a symmetric $(0,2)$-tensor $E$ we define the (0,4)-tensor $\bar{E}$ by $\bar{E}=\frac{1}{2} E \wedge E$. We have $\bar{g}=G=\frac{1}{2} g \wedge g$ and

$$
C=R-\frac{1}{n-2} g \wedge S+\frac{\kappa}{(n-2)(n-1)} G .
$$

We also have (see e.g., $[\mathbf{9}$, Section 3])

$$
Q(E, E \wedge F)=-Q(F, \bar{E}) .
$$

Now (2.2) and (2.3) yield

$$
Q(g, C)=Q(g, R)+\frac{1}{n-2} Q(S, G) .
$$

On any semi-Riemannian manifold $(M, g), n \geqslant 3$, we have $\mathcal{U}_{C} \cap \mathcal{U}_{S} \subset \mathcal{U}_{R} \subset M$.

Let $(M, g), n \geqslant 4$, be a semi-Riemannian manifold such that its curvature tensor $R$ satisfies on $\mathcal{U}_{C} \cap \mathcal{U}_{S} \subset M$ the equation

$$
R=\frac{\phi}{2} S \wedge S+\mu g \wedge S+\eta G
$$

where $\phi, \mu$ and $\eta$ are some functions on this set. According to [6], (2.5) is called the Roter type equation. A manifold $(M, g), n \geqslant 4$, satisfying (2.5) on $\mathcal{U}_{C} \cap \mathcal{U}_{S} \subset M$ will be called a Roter type manifold. Evidently, we consider manifolds $(M, g)$ with nonempty set $\mathcal{U}_{C} \cap \mathcal{U}_{S} \subset M$. The decomposition of $R$ on $\mathcal{U}_{C} \cap \mathcal{U}_{S}$ in terms $S \wedge S$, $g \wedge S$ and $G$ is unique [12, Lemma 3.2]. If (2.5) holds on an open set $U \subset \mathcal{U}_{C} \cap \mathcal{U}_{S}$, then we say that the Roter type equation holds on $U$. Roter type manifolds were defined in [6], although investigations on these manifolds were initiated earlier in [11]. If $(M, g)$ is a Roter type manifold, then (1.2) holds on $\mathcal{U}_{C} \cap \mathcal{U}_{S}$, with the function $L_{R}$ defined by $L_{R}=(n-2)\left(\frac{\mu}{\phi}\left(\mu-\frac{1}{n-2}\right)-\eta\right)$ [11, Theorem 4.2]. If (1.1) and $(2.5)$ hold at a point of a semi-Riemannian manifold of dimension $\geqslant 4$, then its Weyl tensor $C$ vanishes at this point.

For a symmetric $(0,2)$-tensor $E$ and a $(0, k)$-tensor $T, k \geqslant 2$, we define their Kulkarni-Nomizu product $E \wedge T$ by [7]

$$
\begin{aligned}
& (E \wedge T)\left(X_{1}, X_{2}, X_{3}, X_{4} ; Y_{3}, \ldots, Y_{k}\right) \\
& =E\left(X_{1}, X_{4}\right) T\left(X_{2}, X_{3}, Y_{3}, \ldots, Y_{k}\right)+E\left(X_{2}, X_{3}\right) T\left(X_{1}, X_{4}, Y_{3}, \ldots, Y_{k}\right) \\
& -E\left(X_{1}, X_{3}\right) T\left(X_{2}, X_{4}, Y_{3}, \ldots, Y_{k}\right)-E\left(X_{2}, X_{4}\right) T\left(X_{1}, X_{3}, Y_{3}, \ldots, Y_{k}\right) \text {. }
\end{aligned}
$$

Using the above definitions we can prove

Lemma 2.1. [7], [8] Let $E_{1}, E_{2}$ and $F$ be symmetric (0,2)-tensors at a point $x$ of a semi-Riemannian manifold $(M, g), n \geqslant 3$. Then at $x$ we have

$$
E_{1} \wedge Q\left(E_{2}, F\right)+E_{2} \wedge Q\left(E_{1}, F\right)=-Q\left(F, E_{1} \wedge E_{2}\right) .
$$

If $E=E_{1}=E_{2}$, then

$$
E \wedge Q(E, F)=-Q(F, \bar{E})
$$


Proposition 2.1. [18, eq. (13)] On any semi-Riemannian manifold $(M, g)$, $n \geqslant 4$, the tensor $C \cdot R$ satisfies the identity

$$
\begin{aligned}
(n-2)(C & \cdot R)_{h i j k l m}=(n-2)(R \cdot R)_{h i j k l m}+Q\left(\frac{\kappa}{n-1} g-S, R\right)_{h i j k l m} \\
& -g_{h l} S_{m}{ }^{r} R_{r i j k}+g_{h m} S_{l}{ }^{r} R_{r i j k}+g_{i l} S_{m}{ }^{r} R_{r h j k}-g_{i m} S_{l}{ }^{r} R_{r h j k} \\
& -g_{j l} S_{m}{ }^{r} R_{r k h i}+g_{j m} S_{l}{ }^{r} R_{r k h i}+g_{k l} S_{m}{ }^{r} R_{r j h i}-g_{k m} S_{l}{ }^{r} R_{r j h i} .
\end{aligned}
$$

\section{Hypersurfaces in spaces of constant curvature}

Let $M, n \geqslant 3$, be a connected hypersurface isometrically immersed in a semiRiemannian manifold $\left(N, g^{N}\right)$. We denote by $g$ the metric tensor induced on $M$ from $g^{N}$. Further, we denote by $\nabla$ and $\nabla^{N}$ the Levi-Civita connections corresponding to the metric tensors $g$ and $g^{N}$, respectively. Let $\xi$ be a local unit normal vector field on $M$ in $N$ and let $\varepsilon=g^{N}(\xi, \xi)= \pm 1$. The Gauss formula and the Weingarten formula of $(M, g)$ in $\left(N, g^{N}\right)$ are given by $\nabla_{X}^{N} Y=\nabla_{X} Y+\varepsilon H(X, Y) \xi$ and $\nabla_{X}^{N} \xi=-\mathcal{A} X$, respectively, where $X, Y$ are vector fields tangent to $M, H$ is the second fundamental tensor of $(M, g)$ in $\left(N, g^{N}\right), \mathcal{A}$ is the shape operator, $H^{k}(X, Y)=g\left(\mathcal{A}^{k} X, Y\right), k \geqslant 1, H^{1}=H$ and $\mathcal{A}^{1}=\mathcal{A}$. We denote by $R$ and $R^{N}$ the Riemann-Christoffel curvature tensors of $(M, g)$ and $\left(N, g^{N}\right)$, respectively.

Let $x^{r}=x^{r}\left(y^{k}\right)$ be the local parametric expression of $(M, g)$ in $\left(N, g^{N}\right)$, where $y^{k}$ and $x^{r}$ are local coordinates of $M$ and $N$, respectively, and $h, i, j, k \in$ $\{1,2, \ldots, n\}$ and $p, r, t, u \in\{1,2, \ldots, n+1\}$. The Gauss equation of $(M, g)$ in $\left(N, g^{N}\right)$ has the form

$$
R_{h i j k}=R_{p r t u}^{N} B_{h}^{p} B_{i}^{r} B_{j}{ }^{t} B_{k}{ }^{u}+\varepsilon\left(H_{h k} H_{i j}-H_{h j} H_{i k}\right), \quad B_{k}{ }^{r}=\frac{\partial x^{r}}{\partial y^{k}},
$$

where $R_{p r t u}^{N}, R_{h i j k}$ and $H_{h k}$ are the local components of the tensors $R^{N}, R$ and $H$, respectively.

Let now $M$ be a hypersurface in $N_{s}^{n+1}(c), n \geqslant 4$. The Gauss equation (3.1) reads

$$
R_{h i j k}=\varepsilon \bar{H}_{h i j k}+\frac{\widetilde{\kappa}}{n(n+1)} G_{h i j k} .
$$

Contracting (3.2) with $g^{i j}$ and $g^{k h}$, respectively, we obtain

$$
\begin{aligned}
S_{h k} & =\varepsilon\left(\operatorname{tr}(H) H_{h k}-H_{h k}^{2}\right)+\frac{(n-1) \widetilde{\kappa}}{n(n+1)} g_{h k}, \\
\kappa & =\varepsilon\left((\operatorname{tr}(H))^{2}-\operatorname{tr}\left(H^{2}\right)\right)+\frac{(n-1) \widetilde{\kappa}}{n+1},
\end{aligned}
$$

respectively, where $\operatorname{tr}(H)=g^{h k} H_{h k}, \operatorname{tr}\left(H^{2}\right)=g^{h k} H_{h k}^{2}$ and $S_{h k}$ are the local components of the Ricci tensor $S$ of $M$. On every hypersurface $M$ in $N_{s}^{n+1}(c), n \geqslant 4$, we have [15]

$$
R \cdot R-Q(S, R)=-\frac{(n-2) \widetilde{\kappa}}{n(n+1)} Q(g, C)
$$


which by making use of (2.4) and (2.6) turns into

$$
R \cdot R=Q(S, R)-\frac{(n-2) \widetilde{\kappa}}{n(n+1)} Q(g, R)-\frac{\widetilde{\kappa}}{n(n+1)} Q(S, G) .
$$

It is known that $\mathcal{U}_{H} \subset \mathcal{U}_{C} \cap \mathcal{U}_{S} \subset M$ (see e.g., [10, Section 2]).

Proposition 3.1. Let $M$ be a hypersurface in $N_{s}^{n+1}(c), n \geqslant 4$. Then (1.4) is equivalent on $U_{1} \subset \mathcal{U}_{H} \subset M$ to

$$
\begin{aligned}
(\varepsilon \psi & -\frac{(n-1) \widetilde{\kappa}}{n(n+1)} Q(g, R)_{h i j k l m}+\frac{\widetilde{\kappa}}{n(n+1)} Q(S, G)_{h i j k l m} \\
= & -g_{h l} S_{m}{ }^{r} R_{r i j k}+g_{h m} S_{l}{ }^{r} R_{r i j k}+g_{i l} S_{m}{ }^{r} R_{r h j k}-g_{i m} S_{l}{ }^{r} R_{r h j k} \\
& -g_{j l} S_{m}{ }^{r} R_{r k h i}+g_{j m} S_{l}{ }^{r} R_{r k h i}+g_{k l} S_{m}{ }^{r} R_{r j h i}-g_{k m} S_{l}{ }^{r} R_{r j h i} .
\end{aligned}
$$

Proof. As it was mentioned in Section 1, (1.4) is equivalent on $U_{1}$ to (1.6). Now (1.6), by making use of (2.7) and (3.4), is equivalent on $U_{1}$ to (3.5), which completes the proof.

Proposition 3.2. Let $M$ be a hypersurface in $N_{s}^{n+1}(c), n \geqslant 4$, and let (1.4) be satisfied on $\mathcal{U}_{H} \subset M$. Then

(i) $O n \mathcal{U}_{H}$ we have

$$
\begin{aligned}
S_{h}{ }^{r} R_{r i j k}= & \left(\frac{(n-1) \widetilde{\kappa}}{n(n+1)}-\varepsilon \psi\right)\left(R_{h i j k}-\frac{\widetilde{\kappa}}{n(n+1)} G_{h i j k}\right) \\
& +\frac{\widetilde{\kappa}}{n(n+1)}\left(g_{i j} S_{h k}-g_{i k} S_{h j}\right)-\rho\left(g_{h k} H_{i j}-g_{h j} H_{i k}\right), \\
(R \cdot S)_{h i j k}= & \frac{\widetilde{\kappa}}{n(n+1)} Q(g, S)_{h i j k}+\rho Q(g, H)_{h i j k}, \\
S^{r s} R_{r i j s}= & \left(\frac{(n-1) \widetilde{\kappa}}{n(n+1)}-\varepsilon \psi\right)\left(S_{i j}-\frac{(n-1) \widetilde{\kappa}}{n(n+1)} g_{i j}\right) \\
& +\frac{\widetilde{\kappa}}{n(n+1)}\left(\kappa g_{i j}-S_{i j}\right)-(n-1) \rho H_{i j}, \\
S_{h k}^{2}= & \left(\frac{2(n-1) \widetilde{\kappa}}{n(n+1)}-\varepsilon \psi\right) S_{h k}+\rho H_{h k} \\
& -\left(\left(\frac{(n-1) \widetilde{\kappa}}{n(n+1)}-\varepsilon \psi\right) \frac{(n-1) \widetilde{\kappa}}{n(n+1)}+\rho \operatorname{tr}(H)\right) g_{h k},
\end{aligned}
$$

$$
\begin{aligned}
S_{h k}^{3} & =\left(\frac{3(n-1) \widetilde{\kappa}}{n(n+1)}-2 \varepsilon \psi\right) S_{h k}^{2} \\
& -\left(\left(\frac{(n-1) \widetilde{\kappa}}{n(n+1)}-\varepsilon \psi\right)\left(\frac{3(n-1) \widetilde{\kappa}}{n(n+1)}-2 \varepsilon \psi\right)+\rho \operatorname{tr}(H)\right) S_{h k} \\
& +\left(\left(\frac{(n-1) \widetilde{\kappa}}{n(n+1)}-\varepsilon \psi\right)\left(\left(\frac{(n-1) \widetilde{\kappa}}{n(n+1)}-\varepsilon \psi\right) \frac{(n-1) \widetilde{\kappa}}{n(n+1)}+\rho \operatorname{tr}(H)\right)-\varepsilon \rho^{2}\right) g_{h k} .
\end{aligned}
$$


(ii) If on $\mathcal{U}_{H}$ we have

$$
\begin{aligned}
S_{h}{ }^{r} R_{\text {rijk }}= & \left(\frac{(n-1) \widetilde{\kappa}}{n(n+1)}-\varepsilon \psi\right)\left(R_{h i j k}-\frac{\widetilde{\kappa}}{n(n+1)} G_{h i j k}\right) \\
& +\frac{\widetilde{\kappa}}{n(n+1)}\left(g_{i j} S_{h k}-g_{i k} S_{h j}\right)+g_{h k} A_{i j}-g_{h j} A_{i k}
\end{aligned}
$$

where $A_{i j}$ are the local components of an arbitrary symmetric (0,2)-tensor $A$ on $\mathcal{U}_{H}$, then (3.5) holds on this set.

Proof. (i) The relations (1.4), (3.2) and (3.3) yield (3.6). From (3.6), by symmetrization in $h, i$ we find (3.7). Contracting (3.6) with $g^{h k}$ we obtain (3.8). Similarly, (3.6) implies (3.9). Further, transvecting (3.9) with $g^{h i} S_{i j}=S^{h}{ }_{j}$, we get

$$
\begin{aligned}
S_{j k}^{3}= & \left(\frac{2(n-1) \widetilde{\kappa}}{n(n+1)}-\varepsilon \psi\right) S_{j k}^{2}+\rho S^{h}{ }_{j} H_{h k} \\
& -\left(\left(\frac{(n-1) \widetilde{\kappa}}{n(n+1)}-\varepsilon \psi\right) \frac{(n-1) \widetilde{\kappa}}{n(n+1)}+\rho \operatorname{tr}(H)\right) S_{j k} .
\end{aligned}
$$

On the other hand, transvecting (3.2) with $g^{h i} H_{i j}=H^{h}{ }_{j}$ and using (1.4), we obtain

$$
H^{h}{ }_{j} S_{h k}=\left(\frac{(n-1) \widetilde{\kappa}}{n(n+1)}-\varepsilon \psi\right) H_{h k}-\varepsilon \rho g_{j k} .
$$

Unbalanced paren- This, by multiplication by $\rho$ and an application of (3.9), yields theses!

$$
\begin{aligned}
\rho H^{h}{ }_{j} S_{h k}= & \left(\frac{(n-1) \widetilde{\kappa}}{n(n+1)}-\varepsilon \psi\right){ }_{?}\left(S_{j k}^{2}-\left(\frac{2(n-1) \widetilde{\kappa}}{n(n+1)}-\varepsilon \psi\right) S_{j k}\right. \\
& \left.+\left(\left(\frac{(n-1) \widetilde{\kappa}}{n(n+1)}-\varepsilon \psi\right) \frac{(n-1) \widetilde{\kappa}}{n(n+1)}+\rho \operatorname{tr}(H)\right) g_{j k}\right)-\varepsilon \rho^{2} g_{j k} .
\end{aligned}
$$

Substituting this into (3.12) we obtain (3.10).

(ii) Using (3.11) we can check that the tensor

$$
\begin{aligned}
& -\left(\varepsilon \psi-\frac{(n-1) \widetilde{\kappa}}{n(n+1)}\right) Q(g, R)_{h i j k l m}-\frac{\widetilde{\kappa}}{n(n+1)} Q(S, G)_{h i j k l m} \\
- & g_{h l} S_{m}{ }^{r} R_{r i j k}+g_{h m} S_{l}{ }^{r} R_{r i j k}+g_{i l} S_{m}{ }^{r} R_{r h j k}-g_{i m} S_{l}{ }^{r} R_{r h j k} \\
- & g_{j l} S_{m}{ }^{r} R_{r k h i}+g_{j m} S_{l}{ }^{r} R_{r k h i}+g_{k l} S_{m}{ }^{r} R_{r j h i}-g_{k m} S_{l}{ }^{r} R_{r j h i}
\end{aligned}
$$

vanishes on $\mathcal{U}_{H}$, which completes the proof.

The last two propositions imply

THEOREM 3.1. Let $M$ be a hypersurface in $N_{s}^{n+1}(c), n \geqslant 4$. Then (1.4) and (3.6) are equivalent on $U_{1} \subset \mathcal{U}_{H} \subset M$.

Proof. (1.4), in view of Proposition 3.2, implies (3.6). Let now (3.6) be fulfilled on $U_{1}$. Clearly, (3.6) is a special form of (3.11). Thus in view of Proposition 3.2(ii), (3.11) implies (3.5). Now Proposition 3.1 completes the proof. 
THEOREM 3.2. If $M$ is a hypersurface in $N_{s}^{n+1}(c), n \geqslant 4$, satisfying (1.4) on $U_{1} \subset \mathcal{U}_{H} \subset M$, then on this set we have

$$
\begin{aligned}
& \rho^{2} R=\frac{\varepsilon}{2}\left(S^{2}-\left(\frac{2(n-1) \widetilde{\kappa}}{n(n+1)}-\varepsilon \psi\right) S+\left(\left(\frac{(n-1) \widetilde{\kappa}}{n(n+1)}-\varepsilon \psi\right) \frac{(n-1) \widetilde{\kappa}}{n(n+1)}+\rho \operatorname{tr}(H)\right) g\right) \\
& \wedge\left(S^{2}-\left(\frac{2(n-1) \widetilde{\kappa}}{n(n+1)}-\varepsilon \psi\right) S+\left(\left(\frac{(n-1) \widetilde{\kappa}}{n(n+1)}-\varepsilon \psi\right) \frac{(n-1) \widetilde{\kappa}}{n(n+1)}+\rho \operatorname{tr}(H)\right) g\right)+\frac{\rho^{2} \widetilde{\kappa}}{n(n+1)} G .
\end{aligned}
$$

Proof. Our assertion is an immediate consequence of (3.2) and (3.9).

REMARK 3.1. (i) In view of the last theorem we can state that the curvature tensor $R$ of some semi-Riemannian manifolds $(M, g), n \geqslant 4$, is expressed by a certain subset of $\mathcal{U}_{C} \cap \mathcal{U}_{S} \subset M$ by a linear combination of the tensors: $S^{2} \wedge S^{2}$, $S \wedge S^{2}, g \wedge S^{2}, S \wedge S, g \wedge S$ and $g \wedge g$, i.e., on this set we have

$$
R=\frac{\phi_{1}}{2} S^{2} \wedge S^{2}+\phi_{2} S \wedge S^{2}+\phi_{3} g \wedge S^{2}+\frac{\phi_{4}}{2} S \wedge S+\phi_{5} g \wedge S+\phi_{6} G,
$$

where $\phi_{1}, \ldots, \phi_{6}$ are some functions on this set. Evidently, (2.5) is a special case of (3.13). Manifolds satisfying (3.13) will be investigated in subsequent papers.

(ii) If $M$ is a hypersurface in a semi-Euclidean space $\mathbb{E}_{s}^{n+1}, n \geqslant 4$, then the set $U_{1} \subset \mathcal{U}_{H} \subset M$ consists of all points of $M$ at which $R \cdot S \neq 0$.

Corollary 3.1. If $M$ is a hypersurface in $\mathbb{E}_{s}^{n+1}, n \geqslant 4$, satisfying (1.4) on $U_{1} \subset \mathcal{U}_{H} \subset M$, then on this set we have

$$
\rho^{2} R=\frac{\varepsilon}{2}\left(S^{2}+\varepsilon \psi S+\rho \operatorname{tr}(H) g\right) \wedge\left(S^{2}+\varepsilon \psi S+\rho \operatorname{tr}(H) g\right) .
$$

\section{Warped product hypersurfaces}

Hypersurfaces $M$ in $N_{s}^{n+1}(c), n \geqslant 4$, which are locally warped products, and in addition, satisfying some curvature conditions on $\mathcal{U}_{H} \subset M$ were investigated in [7] and [8], see e.g., Theorem 4.2 of [7]. It is easy to see that without loss of generality the assumptions of that theorem: $n \geqslant 5$ and $n-p=\operatorname{dim} \widetilde{N} \geqslant 4$, respectively, can be replaced by the assumption $n \geqslant 4$ and $n-p=\operatorname{dim} \tilde{N} \geqslant 3$, respectively. Thus we have

TheOREM 4.1. [7, Theorem 4.2(i)] Let $M$ be a hypersurface in a semi-Euclidean space $\mathbb{E}_{s}^{n+1}, n \geqslant 4$, and let $g$ be the metric induced on $M$ from the metric tensor of $\mathbb{E}_{s}^{n+1}$. Let $U \subset \mathcal{U}_{H} \subset M$ be an open submanifold of $M$ such that $(U, g)=\bar{M} \times_{F} \tilde{N}$, where $(\bar{M}, \bar{g}), p=\operatorname{dim} \bar{M} \geqslant 1$ and $(\widetilde{N}, \widetilde{g}), n-p=\operatorname{dim} \widetilde{N} \geqslant 3$, are some semiRiemannian manifolds and $F$ is the warping function. Let $x$ be a point of $U$ at which the tensors $R \cdot R$ and $Z(\widetilde{R})$ are nonzero and let $V \subset U$ be a coordinate neighbourhood of $x$ such that the tensors $R \cdot R$ and $Z(\widetilde{R})$ are nonzero at every point of $V$. 
The following relations are fulfilled on $V$

$$
\begin{gathered}
\text { (a) } \bar{R}_{a b c d}=0, \quad \text { (b) } T_{a d}=0, \quad \text { (c) } \frac{\Delta_{1} F}{4 F}=c_{0}=\text { const }, \\
(d) \kappa=\frac{1}{F}\left(\kappa_{2}-(n-p)(n-p-1) c_{0}\right),
\end{gathered}
$$

where $\kappa_{2}$ is the scalar curvature of $(\tilde{N}, \widetilde{g})$.

In the following we use notations from [7]. We have

THEOREM 4.2. Let $M$ be a hypersurface in a semi-Euclidean space $\mathbb{E}_{s}^{n+1}, n \geqslant 4$, satisfying (1.4) on $\mathcal{U}_{H} \subset M$. Moreover, let $V \subset \mathcal{U}_{H}$ be the set defined in Theorem 4.1. If the assumptions of Theorem 4.1 are satisfied, then on $V$ we have

$$
\rho=0 \text {. }
$$

Proof. Let $H_{i j}$ be the local components of the second fundamental tensor $H$ of $M$. Thus (1.4) reads

$$
H_{i j}^{3}=\operatorname{tr}(H) H_{i j}^{2}+\psi H_{i j}+\rho g_{i j}
$$

On $V$ we have (see the proof of Theorem 4.2 of $[7]) H_{b c}=0$, where $b, c \in$ $\{1,2, \ldots, p\}$. Therefore (4.2) reduces to $0=\rho g_{b c}=\rho \bar{g}_{b c}$, whence it follows (4.1), completing the proof.

Further, we have

THEOREM 4.3. Let $M$ be a hypersurface in $N_{s}^{n+1}(c), n \geqslant 4, c \neq 0$ and let $g$ be the metric induced on $M$ from the metric tensor of the ambient space. Let $U \subset \mathcal{U}_{H} \subset M$ be an open submanifold of $M$ such that $(U, g)=\bar{M} \times_{F} \tilde{N}$, where $(\bar{M}, \bar{g}), p=\operatorname{dim} \bar{M} \geqslant 1$ and $(\tilde{N}, \widetilde{g}), n-p=\operatorname{dim} \tilde{N} \geqslant 4$, are some semi-Riemannian manifolds and $F$ is the warping function. Let $x$ be a point of $U$ at which the tensors $R \cdot R$ and $Z(\widetilde{R})$ are nonzero and let $V \subset U$ be a coordinate neighbourhood of $x$ such that the tensors $R \cdot R$ and $Z(\widetilde{R})$ are nonzero at every point of $V$.

(i) The following relations are fulfilled on $V$

(a) $H_{a d}=0$,

(b) $T_{a d}=-\frac{2 \widetilde{\kappa} F}{n(n+1)} g_{a d}, \quad \operatorname{tr}(T)=-\frac{2 p \widetilde{\kappa} F}{n(n+1)}$,

(c) $\frac{\Delta_{1} F}{4 F}=c_{0}-\frac{F \widetilde{\kappa}}{n(n+1)}, \quad c_{0}=$ const .

$$
\text { (d) } \kappa=\kappa_{1}+\frac{\kappa_{2}}{F}+\frac{1}{F}\left(\frac{(n-p)(n+p-1) \widetilde{\kappa}}{n(n+1)}-(n-p)(n-p-1) c_{0}\right) \text {, }
$$

where $\kappa_{1}$ and $\kappa_{2}$ are the scalar curvatures of $(\bar{M}, \bar{g})$ and $(\tilde{N}, \widetilde{g})$, respectively. In addition, if $p \geqslant 2$, then

$$
\text { (a) } \quad \bar{R}_{a b c d}=\frac{\kappa_{1}}{p(p-1)} G_{a b c d}, \quad \text { (b) } \quad \frac{\kappa_{1}}{p(p-1)}=\frac{\widetilde{\kappa}}{n(n+1)}
$$


(ii) The local components of the curvature tensor $R$ and the Ricci tensor $S$ of $(U, g)$ and the second fundamental tensor $H$ of $U$ in $M$ which may not vanish identically on $V$ are the following

$$
\begin{aligned}
\text { (a) } R_{\alpha \beta \gamma \delta} & =\varepsilon F\left(\widetilde{H}_{\alpha \delta} \widetilde{H}_{\beta \gamma}-\widetilde{H}_{\alpha \gamma} \widetilde{H}_{\beta \delta}\right)+\frac{\widetilde{\kappa}}{n(n+1)} G_{\alpha \beta \gamma \delta}, \\
\text { (b) } \widetilde{H}_{\alpha \delta} & =\widetilde{H}_{\alpha \delta}\left(x^{p+1}, \ldots, x^{n}\right), \\
S_{\alpha \beta} & =\widetilde{S}_{\alpha \beta}+\left(\frac{(n-1) \widetilde{\kappa} F}{n(n+1)}-(n-p-1) c_{0}\right) \widetilde{g}_{\alpha \beta}, \\
\text { (a) } H_{\alpha \delta} & =\sqrt{F} \widetilde{H}_{\alpha \delta}, \quad \text { (b) } \widetilde{\nabla}_{\alpha} \widetilde{H}_{\beta \delta}=\widetilde{\nabla}_{\beta} \widetilde{H}_{\alpha \delta} .
\end{aligned}
$$

(iii) We have on $V$

$$
(\widetilde{R} \cdot \widetilde{R})_{\alpha \beta \gamma \delta \epsilon \mu}-Q(\widetilde{S}, \widetilde{R})_{\alpha \beta \gamma \delta \epsilon \mu}=-(n-p-2) c_{0} Q(\widetilde{g}, \widetilde{C})_{\alpha \beta \gamma \delta \epsilon \mu} .
$$

(iv) If (1.4) is satisfied on $V$, then on this set we have $\rho=0$ and (4.8) $\widetilde{S}_{\alpha}{ }^{\mu} \widetilde{R}_{\mu \beta \gamma \delta}=\left((n-p-1) c_{0}-\varepsilon \psi F\right)\left(R_{\alpha \beta \gamma \delta}-c_{0} G_{\alpha \beta \gamma \delta}\right)+c_{0}\left(\widetilde{g}_{\beta \gamma} \widetilde{S}_{\alpha \delta}-\widetilde{g}_{\beta \delta} \widetilde{S}_{\alpha \gamma}\right)$.

Proof. By making use of (8), (9), (10) and (13) of [7] we obtain on $V$ the following relations

$$
\begin{gathered}
\bar{R}_{a b c d}=R_{a b c d}=\varepsilon\left(H_{a d} H_{b c}-H_{a c} H_{b d}\right)+\frac{\widetilde{\kappa}}{n(n+1)} G_{a b c d}, \\
-\frac{1}{2} T_{a d} \widetilde{g}_{\alpha \beta}=R_{a \alpha \beta d}=\varepsilon\left(H_{a d} H_{\alpha \beta}-H_{a \beta} H_{\alpha d}\right)+\frac{\widetilde{\kappa}}{n(n+1)} g_{a d} g_{\alpha \beta}, \\
\begin{aligned}
& F \widetilde{R}_{\alpha \beta \gamma \delta}-\frac{\Delta_{1} F}{4} \widetilde{G}_{\alpha \beta \gamma \delta}=R_{\alpha \beta \gamma \delta} \\
&=\varepsilon\left(H_{\alpha \delta} H_{\beta \gamma}-H_{\alpha \gamma} H_{\beta \delta}\right)+\frac{\widetilde{\kappa}}{n(n+1)} G_{\alpha \beta \gamma \delta}, \\
& 0= R_{a \alpha \beta \gamma}=\varepsilon\left(H_{a \gamma} H_{\alpha \beta}-H_{a \beta} H_{\alpha \gamma}\right), \\
& \text { (a) } S_{a d}=\bar{S}_{a d}-\frac{n-p}{2 F} T_{a b}, \\
& \text { (b) } S_{\alpha \delta}=\widetilde{S}_{\alpha \delta}-\left(\frac{\operatorname{tr}(T)}{2}+(n-p-1) \frac{\Delta_{1} F}{4 F}\right) \widetilde{g}_{\alpha \delta} .
\end{aligned}
\end{gathered}
$$

We note that if all components of the form $H_{\alpha \delta}$ vanish at $y \in V$, then from (4.11) it follows that the tensor $Z(\widetilde{R})$ vanishes at this point, a contradiction. Thus at every point of $V$ at least one of the local components $H_{\alpha \delta}$ must be nonzero. Therefore from (4.12) we can deduce that $H_{a \gamma}=0$ at every point of $V$. Now (4.10) turns into

$$
-\frac{1}{2} T_{a d} \widetilde{g}_{\alpha \beta}=\varepsilon H_{a d} H_{\alpha \beta}+\frac{\widetilde{\kappa} F}{n(n+1)} g_{a d} \widetilde{g}_{\alpha \beta},
$$

whence

$$
-\frac{1}{2} T_{a d}=\frac{\varepsilon}{n-p} \widetilde{g}^{\gamma \delta} H_{\gamma \delta} H_{a d}+\frac{\widetilde{\kappa} F}{n(n+1)} g_{a d}
$$


Substituting this into (4.14) we obtain

$$
H_{a d}\left(H_{\alpha \beta}-\frac{1}{n-p} \widetilde{g}^{\gamma \delta} H_{\gamma \delta} \widetilde{g}_{\alpha \beta}\right)=0 .
$$

If at $y \in V$ all components of the form $H_{\alpha \beta}$ are proportional to $\widetilde{g}_{\alpha \beta}$, then by (4.11) at this point we have $Z(\widetilde{R})=0$, a contradiction. Thus all components of $H$ of the form $H_{a d}$ must vanish at every point of $V$, i.e., (4.3)(a) holds on $V$. Thus (4.14) reduces to (4.3)(b). Clearly, if $p \geqslant 2$, then (4.9) implies (4.4).

Since $H$ is a Codazzi tensor, we have $\nabla_{a} H_{\beta \gamma}=\nabla_{\beta} H_{a \gamma}$ and $\nabla_{\alpha} H_{\beta \gamma}=\nabla_{\beta} H_{\alpha \gamma}$. From these relations, by making use of (7) of [7], we obtain (4.4)(b) and (4.6). Further, (4.11) and (4.6)(a) yield (4.3)(c). Now using (4.3)(b), (4.3)(c), (4.13) and the identity $\kappa=g^{a d} S_{a d}+\frac{1}{F} \widetilde{g}^{\alpha \delta} S_{\alpha \delta}$ we obtain (4.3)(d). (4.7) is a consequence of (19) and (34) of [4], (4.6)(a) and (4.6)(b) and the identity

$$
Q(g, C)_{\alpha \beta \gamma \delta \epsilon \mu}=F^{2}\left(Q(\widetilde{g}, \widetilde{R})_{\alpha \beta \gamma \delta \epsilon \mu}+\frac{1}{n-2} Q(\widetilde{S}, \widetilde{G})_{\alpha \beta \gamma \delta \epsilon \mu}\right) .
$$

In the same manner as in the proof of Theorem 4.2 we can show that $\rho=0$ on $V$. Further, in view of Proposition 3.2, (1.4) implies (3.6). Since $\rho$ vanishes on $V$, (3.6) yields

$$
\begin{aligned}
g^{\epsilon \mu} S_{\alpha \epsilon} R_{\mu \beta \gamma \delta}= & \left(\frac{(n-1) \widetilde{\kappa}}{n(n+1)}-\varepsilon \psi\right)\left(R_{\alpha \beta \gamma \delta}-\frac{\widetilde{\kappa}}{n(n+1)} G_{\alpha \beta \gamma \delta}\right) \\
& +\frac{\widetilde{\kappa}}{n(n+1)}\left(g_{\beta \gamma} S_{\alpha \delta}-g_{\beta \delta} S_{\alpha \gamma}\right) .
\end{aligned}
$$

Applying in this (9) of $[7],(4.3)(\mathrm{b}),(4.3)(\mathrm{c})$ and (4.5), we can check that (4.8) holds on $V$. Our theorem is thus proved.

\section{References}

[1] B.E. Abdalla and F. Dillen, A Ricci-semi-symmetric hypersurface of the Euclidean space which is not semi-symmetric, Proc. Amer. Math. Soc. 130 (2002), 1805-1808.

[2] K. Arslan, R. Deszcz, R. Ezentaș, C. Murathan, and C. Özgür, On pseudosymmetry type hypersurfaces of semi-Euclidean spaces I, Acta Math. Scientia, 22B (2002), 346-358.

[3] M. Belkhelfa, R. Deszcz, M. Głogowska, M. Hotloś, D. Kowalczyk, and L. Verstraelen, On some type of curvature conditions, in: Banach Center Publ. 57, Inst. Math., Polish Acad. Sci., 2002, 179-194.

[4] F. Defever and R. Deszcz, On warped product manifolds satisfying a certain curvature condition, Atti Accad. Peloritana Pericolanti, Cl. Sci. Fis. Mat. Nat. 69 (1991), 213-236.

[5] F. Defever, R. Deszcz, P. Dhooghe, L. Verstraelen, and Ș. Yaprak, On Ricci-pseudosymmetric hypersurfaces in spaces of constant curvature, Results Math. 27 (1995), 227-236.

[6] R. Deszcz, On some Akivis-Goldberg type metrics, Publ. Inst. Math. (Beograd) (N.S.) 74(88) (2003), 71-83.

[7] R. Deszcz and M. Głogowska, Some nonsemisymmetric Ricci-semisymmetric warped product hypersurfaces, Publ. Inst. Math. (Beograd) 72(86) 2002, 81-93.

[8] R. Deszcz and M. Głogowska, Examples of nonsemisymmetric Ricci-semisymmetric hypersurfaces, Colloq. Math. 94 (2002), 87-101.

[9] R. Deszcz, M. Głogowska, M. Hotloś, and Z. Șentürk, On certain quasi-Einstein semisymmetric hypersurfaces, Ann. Univ. Sci. Budap. Rolando Eötvös, Sect. Math. 41 (1998), 151-164. 
[10] R. Deszcz, M. Głogowska, M. Hotloś, and L. Verstraelen, On some generalized Einstein metric conditions on hypersurfaces in semi-Riemannian space forms, Colloq. Math. 96 (2003), 149166.

[11] R. Deszcz and M. Hotloś, On a certain subclass of pseudosymmetric manifolds, Publ. Math. Debrecen 53 (1998), 29-48.

[12] R. Deszcz and M. Hotloś, On hypersurfaces with type number two in space forms, Ann. Univ. Sci. Budap. Rolando Eötvös, Sect. Math., 46 (2003), 19-34.

[13] R. Deszcz and D. Kowalczyk, On some class of pseudosymmetric warped products, Colloq. Math. 97 (2003), 7-22.

[14] R. Deszcz and K. Sawicz, On some class of hypersurfaces in Euclidean spaces, Ann. Univ. Sci. Budap. Rolando Eötvös, Sect. Math., in print.

[15] R. Deszcz and L. Verstraelen, Hypersurfaces of semi-Riemannian conformally flat manifolds, in: Geometry and Topology of Submanifolds, III, World Sci., River Edge, NJ, 1991, 131-147.

[16] M. Głogowska, On a curvature characterization of Ricci-pseudosymmetric hypersurfaces, Acta Math. Scientia, 24B (2004), 361-375.

[17] M. Głogowska, Curvature conditions on hypersurfaces with two distinct principal curvatures, in: Banach Center Publ. 69, Inst. Math., Polish Acad. Sci., 2005, 133-143.

[18] C. Murathan, K. Arslan, R. Deszcz, R. Ezentaș, and C. Özgür, On a certain class of hypersurfaces of semi-Euclidean spaces, Publ. Math. Debrecen 58 (2001), 587-604.

[19] K. Sawicz, Hypersurfaces in spaces of constant curvature satisfying some Ricci-type equations, Colloq. Math. 101 (2004), 183-201.

[20] K. Sawicz, On some class of hypersurfaces with three principal curvatures, in: Banach Center Publ. 69, Inst. Math., Polish Acad. Sci., 2005, 145-156.

[21] K. Sawicz, Examples of hypersurfaces in Euclidean spaces with three principal curvatures, Dept. Math. Agricultural Univ. Wrocław, Ser. A, Theory and Methods, Report No. 114, 2005.

[22] Z. I. Szabó, Structure theorems on Riemannian spaces satisfying $R(X, Y) \cdot R=0$. I. The local version, J. Differential Geom. 17 (1982), 531-582.

Institute of Econometrics and Computer Science

(Received 1201 2006)

Technical University of Czestochowa

Armii Krajowej 19B

42-200 Czestochowa

Poland

ksawicz@zim.pcz.czest.pl 